\title{
OPEN Diagnostic accuracy of the 1,3-beta-D-glucan test and lactate dehydrogenase for pneumocystis pneumonia in non-HIV patients
}

\author{
Ruixue Sun ${ }^{1}$, Dan $\mathrm{Lv}^{2}$, Meng Xiao ${ }^{3}$, Li Zhang ${ }^{3}$, Jun $\mathrm{Xu}^{1}$, Xuezhong Yu ${ }^{1}$, Huadong Zhu ${ }^{1}$ \& \\ Jing Yang ${ }^{1 \bowtie}$
}

We evaluated the serum levels of (1-3)-beta-D-glucan (BG) and lactate dehydrogenase (LDH) as a tool to support pneumocystis pneumonia (PCP) diagnostic procedures in non-HIV patients. We retrospectively collected non-HIV (human immunodeficiency virus) patients presenting clinical features of PCP between April 1st, 2013, and December 31st, 2018. A total of 225 included patients were tested for Pneumocystis jirovecii by polymerase chain reaction (PCR) and methenamine silver staining. Based on different exclusion criteria, 179 cases were included in the BG group, and 196 cases were included in the LDH group. In each group, cases with positive immunofluorescence (IF) microscopy and $P C R$ were considered proven $P C P$, while cases with only positive $P C R$ were considered probable PCP. Fifty patients with negative IF and PCR results and proven to be non-PCP infection were chosen randomly as the control group. The cut-off levels of BG and LDH to distinguish non-PCP from probable PCP were $110 \mathrm{pg} / \mathrm{mL}$ and $296 \mathrm{U} / \mathrm{L}$ with $88 \%$ sensitivity and $86 \%$ specificity, and $66 \%$ sensitivity and $88 \%$ specificity, respectively. The cut-off levels of BG and LDH to distinguish non-PCP from proven PCP were $285.8 \mathrm{pg} / \mathrm{mL}$ and $379 \mathrm{U} / \mathrm{L}$ with $92 \%$ sensitivity and $96 \%$ specificity, and $85 \%$ sensitivity and $77 \%$ specificity, respectively. The cut-off levels of BG and LDH to distinguish nonPCP from proven/probable PCP were $144.1 \mathrm{pg} / \mathrm{mL}$ and $363 \mathrm{U} / \mathrm{L}$ with $90 \%$ sensitivity, $86 \%$ specificity and $80 \%$ sensitivity, $76 \%$ specificity respectively. BG and LDH are reliable indicators for detecting $P$. jirovecii infection in HIV-uninfected immunocompromised patients.

Pneumocystis pneumonia (PCP) is a potentially life-threatening infection that occurs in immunocompromised patients. HIV-infected patients are at the highest risk of PCP. In our hospital, nearly half of the population who at high risk of PCP infection are patients with rheumatic immune diseases, because they have to receive long-term treatment with glucocorticoids and other immunosuppressive drugs. As reported, other non-HIV immunocompromised patients include haematopoietic stem cell recipients, solid organ transplant recipients and patients with cancer ${ }^{1}$. As PCP causes serious morbidity, timely diagnosis means better prognosis for these patients ${ }^{2}$.

Non-HIV immunosuppressed patients with PCP typically present with hypoxaemia associated with fever, dry cough and significant dyspnoea ${ }^{1,3}$. The radiographic findings in PCP are nonspecific; in some patients, HRCT may reveal an extensive 'ground-glass' appearance or cystic lesions. Although clinical and radiographic findings can be highly suggestive of a diagnosis of PCP in immunocompromised patients, the definitive diagnosis of PCP requires identification of the organism either by tinctorial staining, fluorescent antibody staining, or polymerase chain reaction (PCR)-based assays of respiratory specimens ${ }^{3}$. However, as patients normally have a dry cough, respiratory specimens should be collected in specific infectious disease clinics or obtained by bronchoalveolar lavage.

\footnotetext{
${ }^{1}$ Emergency Department, State Key Laboratory of Complex Severe and Rare Diseases, Peking Union Medical College Hospital, Chinese Academy of Medical Science and Peking Union Medical College, Beijing, China. ${ }^{2}$ Beijing Emergency Medical Centre, Beijing, China. ${ }^{3}$ Laboratory Department, State Key Laboratory of Complex Severe and Rare Diseases, Peking Union Medical College Hospital, Chinese Academy of Medical Science and Peking Union Medical College, Beijing, China. ${ }^{\circledR}$ email: pumchyangjing@126.com
} 
These two methods are difficult to implement. Thus, the 1,3-beta-D-glucan (BG) test and lactate dehydrogenase $(\mathrm{LDH})$, which are more readily available by blood tests, may be used to help support the diagnosis of $\mathrm{PCP}^{3,4}$.

Beta-D-glucan is the major component of $P$. jirovecii's cyst wall ${ }^{5}$. Several studies have demonstrated that a high level of BG is a discriminative marker of PCP in immunocompromised patients, but the target population is HIV-infected patients ${ }^{5-9}$ or HIV-infected patients mixed with non-HIV-infected patients ${ }^{10-13}$. Only a few studies have merely focused on non-HIV-infected patients, and the main underlying conditions of these patients are malignancy or solid organ transplantation ${ }^{8,14}$. However, prospective data about the use of BG for diagnosing PCP in non-HIV-uninfected patients are limited. As HIV-related PCP and non-HIV-related PCP have different clinical characteristics and prognoses ${ }^{15,16}$, whether the observations related to the accuracy of BG as a diagnostic marker for PCP in HIV-infected individuals can be extrapolated to the non-HIV population is still a question. In addition, $\mathrm{LDH}$ is regarded as a non-specific marker of PCP because it is possibly caused by the underlying lung inflammatory responses and damage ${ }^{3}$. In Deng C's study, the sensitivity of LDH in the diagnosis of PCP was above $80 \%$ in the HIV group ${ }^{17}$. We are interested in its sensitivity and specificity in the HIV-negative group. In our study, we explored the ability of serum BG and LDH to support the diagnosis of PCP in non-HIV conditions.

\section{Methods}

Study design. Peking Union Medical College Hospital receives hundreds of non-HIV immunocompromised patients from mainland China. We conducted a retrospective study in our hospital. All patients admitted with a diagnosis of PCP from April 1st, 2013, to December 31st, 2018, were screened for eligibility. The inclusion criteria were as follows: (1) presenting one or more of the following clinical features: fever, dry cough, hypoxaemia, and CT scan demonstrating bilateral infiltrates or ground glass; (2) respiratory secretions were tested by methenamine silver staining and polymerase chain reaction (PCR) to detect P. jirovecii; and (3) HIV tests were negative. The exclusion criteria were as follows: (1) patients received therapeutic doses of empiric treatment for PCP for over 3 days before sample collection to perform PCP IF/PCR tests; (2) sera for BG and LDH tests were collected 7 days from sample collection to perform PCP IF/PCR; (3) patients with non-PCP fungal infection or those undergoing therapies may have increased serum BG levels (such as albumin, intravenous immunoglobin, haemodialysis, etc.) and cannot be included in the BG group; and (4) abnormal liver function or haematological malignancies were not included in the LDH group. In each case group, cases with methenamine silver stain and PCR were considered proven PCP; if only the PCR was positive, they were considered probable PCP. Patients with negative methenamine silver staining and PCR results that proved to be non-PCP infections were chosen randomly as the control group, and they also matched the exclusion criteria of the BG case group and LDH case group (Fig. 1: patient sample flow).

From patient records, we collected patient information, including age, sex, length of hospital stays, ICU stay, risk factors (autoimmune disease, haematological malignancies, solid tumour, organ transplantation, bone marrow transplantation), therapy history (glucocorticoid, immunosuppressant and preventive dose of sulfanilamide), clinical manifestation (fever, cough, wheeze), chest CT features, level of BG and LDH, methenamine silver stain and PCP PCR test results, and prognosis. Finally, the level of BG and the LDH value on PCP diagnosis were analysed, and the clinical features of the cases were summarized at the same time.

Laboratory analyses. Serum BG levels were determined by kinetic chromogenic kit (fungal (1-3)- $\beta$-D glucan detection kit produced by CHARLES RIVER Company, Zhanjiang, China), and the test was performed in duplicate where the normal range of $\mathrm{BG}$ is $<100.5 \mathrm{pg} / \mathrm{mL}$. LDH levels were determined using the quantitative Lactate Dehydrogenase Assay Kit (BECKMAN, USA), where the normal range is 0-250 U/L. The pneumocystis is detected by immunofluorescence microscopy after Grocott methenamine silver stain(GMS). The process of GMS is described below, and IF (the kit was Gold Star Taq Man Mixture provided by CoWin Biosciences) microscopy was performed directly after the GMS. $P$. jirovecii DNA was detected in respiratory secretions by an in-house quantitative real-time PCR. BG and LDH testing was performed in a blinded manner to the IF microscopy and PCR results.

Grocott methenamine stain. Formalin-fixed samples on glass slides were incubated with periodic acid for $10 \mathrm{~min}$, then stained by silver solution for $1.5 \mathrm{~h}$ in a water bath at $56^{\circ} \mathrm{C}$, and stained with $0.25 \%$ gold chloride solution, $3 \%$ sodium thiosulfate, brilliant green by turn for $1 \mathrm{~min}$ respectively, then the result was read. The slides were rinsed with water after every step ${ }^{18}$.

Pneumocystis jirovecii DNA detection. Pneumocystis jirovecii DNA was detected by real-time PCR. The respiratory sample was mixed with $4 \% \mathrm{NaOH}$ solution, and $1 \mathrm{~mL}$ of the liquefaction was placed in an experimental tube and centrifuged at $12,000 \mathrm{rpm} / \mathrm{min}$ for $5 \mathrm{~min}$. Then, the supernatant was discarded. The remaining liquefaction was mixed with normal saline and centrifuged again at the same speed for $1 \mathrm{~min}$, and then the supernatant was discarded. Next, $50 \mu \mathrm{L}$ of magnetic beads (Da An Gene Co., Ltd. of Sun Yat-sen University) was added to the residue and incubated for $5 \mathrm{~min}$ at $100{ }^{\circ} \mathrm{C}$ after oscillating centrifugation, and used for real-time PCR amplification immediately. P. jirovecii-specific primer and fluorescent probe were designed targeting mitochondrial larger subunit (mtLSU) rRNA region, and human albumin gene was used for internal control ${ }^{18}$.

The interpretation of the results is as follows: (1) positive: the fluorescence signal increased significantly, and the amplification curve was S-shaped, with a Ct value $\leq 37$; (2) negative: the fluorescence signal did not increase and showed an S-shape; (3) grey area: the fluorescence signal increased significantly, and the amplification curve was S-shaped, with a Ct value $>37$. The grey area result needs to be repeated; if the confirmatory result is positive or still in the grey area, the final result is weakly positive, and if the confirmatory result is negative, the final 


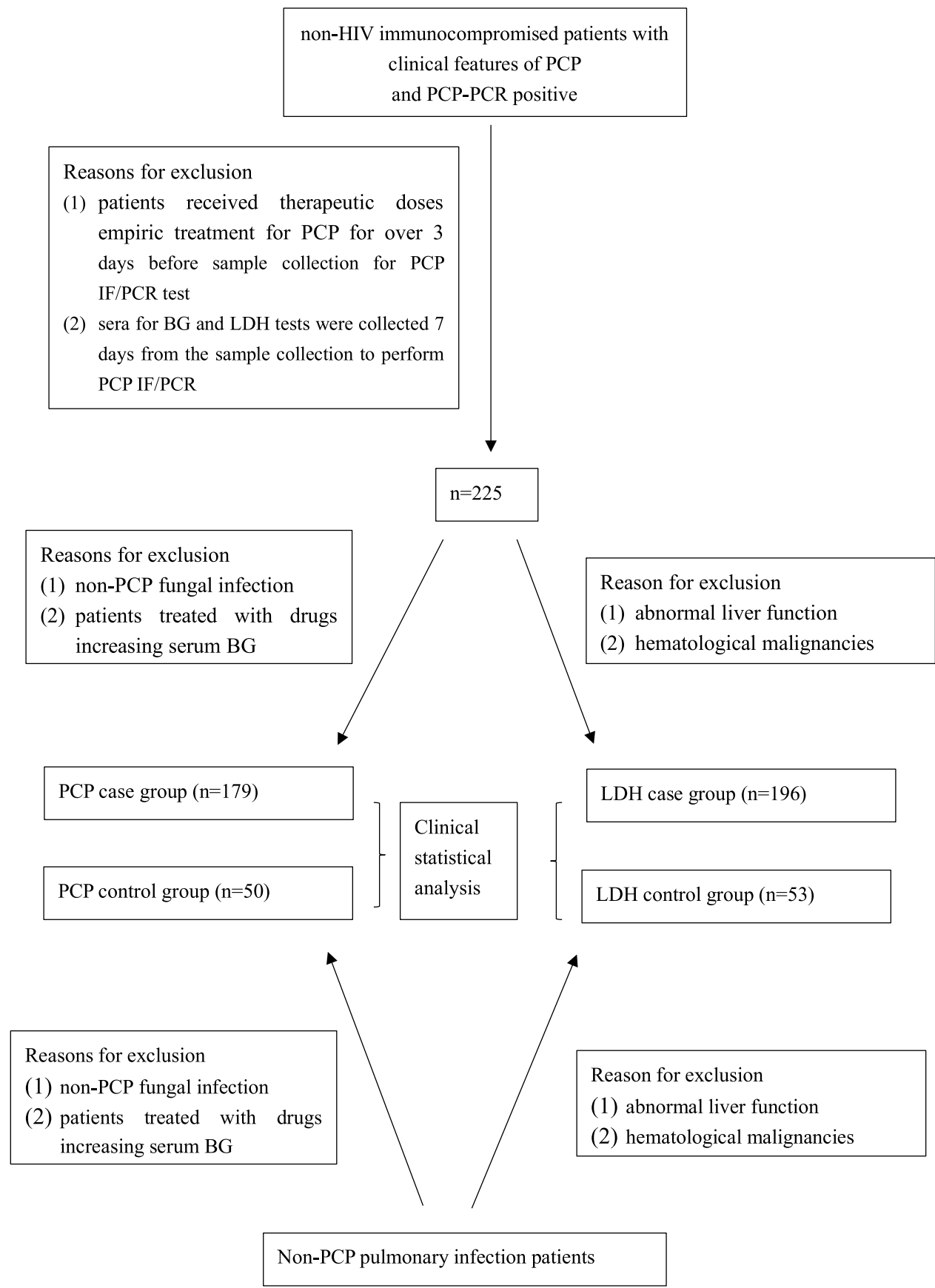

Figure 1. patients sample flow. $H I V$ human immunodeficiency virus, $P C P$ pneumocystis pneumonia, $P C R$ polymerase chain reaction, $I F$ immunofluorescence, $B G(1-3)$-beta-D-glucan, $L D H$ lactate dehydrogenase.

result is negative. In the study, both positive and weekly positive results were treated as "PCP-PCR positive", and a negative result was treated as "PCP-PCR negative" 18 .

Statistics. Numerical variables are provided as the mean (standard deviation [SD]) and median (range, interquartile range $[\mathrm{IQR}]$ ), and categorical data are described by absolute numbers and frequencies. Numerical variables were analysed by the unpaired t-test or Mann-Whitney test for two groups or by one-way ANOVA with post hoc pairwise comparisons by LSD's test for more than two groups. Categorical variables were compared by $\chi^{2}$ or Fisher's exact test. To evaluate test performance, sensitivity and specificity were calculated with $95 \%$ confidence intervals. The positive likelihood ratio (P-LR) was calculated as sensitivity/(1 - specificity), and the negative likelihood ratio (N-LR) was calculated as $(1-$ sensitivity)/specificity with $95 \%$ confidence intervals. The percentage agreement between the test results and Cohen's kappa coefficient $(\mathrm{K})$ was also calculated. For correlation analysis, a scatterplot was constructed, and Spearman's rank correlation coefficient (rs) 


\begin{tabular}{|l|l|l|l|}
\hline & Proven PCP $(\mathbf{n}=24)$ & Probable PCP (n= 155) & Negative PCP (n=50) \\
\hline Male/female ratio & $1: 1$ & $1.21: 1$ & $1.53: 1$ \\
\hline Age (years), media (range) & $50(21-83)$ & $50(15-83)$ & $59(18-84)$ \\
\hline Biological samples type & \multicolumn{5}{|l|}{} \\
\hline Bronchoalveolar lavage fluids (n, \%) & $10(41.67)$ & $40(25.81)$ & $21(42.00)$ \\
\hline Induced sputa (n, \%) & $14(58.33)$ & $115(74.19)$ & $29(58.00)$ \\
\hline Underlying condition & $5(20.83)$ & $18(11.61)$ & - \\
\hline SLE (n, \%) & $10(41.67)$ & $54(34.84)$ & - \\
\hline non-SLE RID (n, \%) & $2(8.33)$ & $8(5.16)$ & - \\
\hline Hematological malignancy (n, \%) & $7(29.17)$ & $75(48.39)$ & - \\
\hline Others (n, \%) & \multicolumn{5}{|l|}{} \\
\hline Outcome of PCP & $25(3-80)$ & $24(2-101)$ & - \\
\hline LOH (days), media (range) & $11(45.83)$ & $78(50.32)$ & - \\
\hline ICU (n, \%) & $6(25.00)$ & $28(18.10)$ & - \\
\hline 30-day mortality, (n, \%) &
\end{tabular}

Table 1. Patient characters of BG groups. SLE systemic lupus erythematosus, RIG rheumatic immunity diseases, $L O H$ length of hospitalization, $I C U$ intensive care unit.

was calculated $^{12}$. Statistics Analysis Software version 9.4 (SAS INSTITUTE INC, Cary, NC, USA) was used at a significance level of 0.05 in Figs. 4 and 5.

Data description and repository. Patient information was collected through the HIS system of Peking Union Medical College Hospital (accession number 11578).

Ethics approval and consent to participate. The Institutional Review Board (IRB) of Peking Union Medical College Hospital reviewed the study and determined that it is a retrospective study and that the design is scientifically sound and meets the ethics standards. The IRB thus approved the study.

Consent for publication. Consent for publication has been obtained from the patients reported in this article.

Statement. All methods in the study were carried out in accordance with relevant guidelines and regulations.

Consent for participate. Informed Consent has been obtained from the patients reported in this article. For patients $<18$ years old, informed Consent was obtained from parents and/or legal guardian.

\section{Result}

The BG group and LDH group included 179 and 196 patients, respectively, and descriptive characteristics are found in Tables 1 and 2. These results showed that the average age of both proven and probable PCP patients was 50 years old, and the male to female ratio was almost 1:1. In addition to systemic lupus erythematosus (SLE) and other rheumatic immune diseases, interstitial lung diseases and glomerulonephritis without definitive cause were common underlying conditions. The percentages of proven and probable PCP patients admitted to ICU were $49.7 \%$ and $46.4 \%$ in BG group and in LDH group respectively. In BG group, the mortality rates of proven PCP patients and probable PCP patients were $25.0 \%$ and $18.1 \%$, and in LDH group, the mortality rates of proven PCP patients and probable PCP patients were $24.2 \%$ and $15.3 \%$.

The mean levels of BG in patients with no PCP, probable PCP and proven PCP were $65.03 \mathrm{pg} / \mathrm{mL}, 983.22 \mathrm{pg} /$ $\mathrm{mL}$, and $1220.09 \mathrm{pg} / \mathrm{mL}$, respectively (Supplementary Table 1 and Fig. 2: The mean levels of BG in the no PCP, probable PCP and proven PCP groups), and the mean levels of LDH in the three groups were 297.62 U/L, 547.88 U/L, and 854.23 U/L (Supplementary Table 2 and Fig. 3: The mean levels of BG in the no PCP, probable PCP and proven PCP groups). Except for the BG level between the probable PCP group and proven PCP group, the levels of BG and LDH between each pair of groups were significantly different (Supplementary Tables 3, 4).

The ROC curves for BG and LDH are shown in Figs. $4 \mathrm{a}-\mathrm{d}$ and $5 \mathrm{a}-\mathrm{d}$. From the analysis of Supplementary Tables 5 and 6, we can see the diagnostic value of BG and LDH for PCP diagnosis: the cut-off levels of BG and LDH to distinguish no PCP from probable PCP were $110 \mathrm{pg} / \mathrm{mL}$ and $296 \mathrm{U} / \mathrm{L}$ with $88 \%$ sensitivity and 86\% specificity, and $66 \%$ sensitivity and $88 \%$ specificity, respectively. The cut-off levels of BG and LDH to distinguish no PCP from proven PCP were $285.8 \mathrm{pg} / \mathrm{mL}$ and $379 \mathrm{U} / \mathrm{L}$ with $92 \%$ sensitivity and $96 \%$ specificity, and $85 \%$ sensitivity and 77\% specificity, respectively. The cut-off levels of BG and LDH to distinguish no PCP from proven/ probable PCP were $144.1 \mathrm{pg} / \mathrm{mL}$ and $363 \mathrm{U} / \mathrm{L}$ with $90 \%$ sensitivity and $86 \%$ specificity, and $80 \%$ sensitivity and $76 \%$ specificity, respectively. However, neither BG nor LDH was good at distinguishing between probable PCP and proven PCP. 


\begin{tabular}{|c|c|c|c|}
\hline & Proven PCP $(n=33)$ & Probable PCP $(n=163)$ & Negative PCP $(n=53)$ \\
\hline Male/female ratio & 1.06:1 & 0.94:1 & $1.44: 1$ \\
\hline Age (years), media (range) & $50(21-72)$ & $50(18-83)$ & $58(18-84)$ \\
\hline \multicolumn{4}{|l|}{ Biological samples type } \\
\hline Bronchoalveolar lavage fluids (n, \%) & $10(30.30)$ & $43(26.38)$ & $23(39.66)$ \\
\hline Induced sputa $(\mathrm{n}, \%)$ & $23(69.70)$ & $120(73.62)$ & $30(60.34)$ \\
\hline \multicolumn{4}{|l|}{ Underlying condition } \\
\hline SLE (n, \%) & $5(15.15)$ & $18(11.04)$ & - \\
\hline non-SLE RID (n, \%) & 11(33.33) & $62(38.04)$ & \\
\hline Others (n, \%) & $17(51.52)$ & $82(50.31)$ & - \\
\hline \multicolumn{4}{|l|}{ Outcome of PCP } \\
\hline LOH (days), media (range) & $33(3-79)$ & $20(2-101)$ & - \\
\hline $\operatorname{ICU}(\mathrm{n}, \%)$ & $14(42.42)$ & $77(42.24)$ & - \\
\hline 30-day mortality, (n, \%) & $8(24.24)$ & $25(15.34)$ & - \\
\hline
\end{tabular}

Table 2. Patient characters of LDH groups.

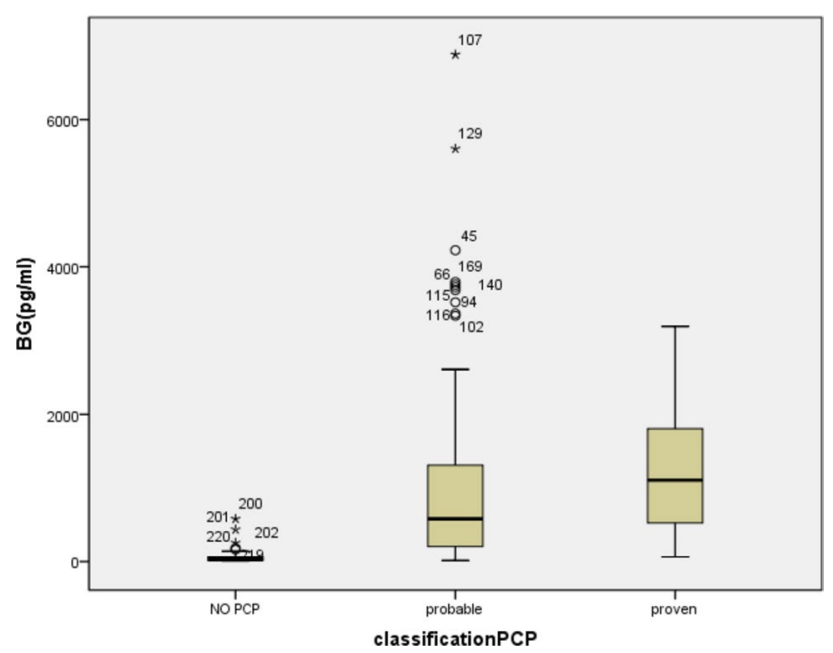

Figure 2. (Box-and-whisker plot): The mean levels of BG in patients with no PCP, probable PCP and proven PCP were $65.03 \mathrm{pg} / \mathrm{mL}, 983.22 \mathrm{pg} / \mathrm{mL}$, and $1220.09 \mathrm{pg} / \mathrm{mL}$, respectively. The medians of the 3 groups were 27.95 $(14.60,65.00) \mathrm{pg} / \mathrm{mL}, 580.0(206.0,1311.0) \mathrm{pg} / \mathrm{mL}, 1107.0(524.1,1806.0) \mathrm{pg} / \mathrm{mL}$ respectively.

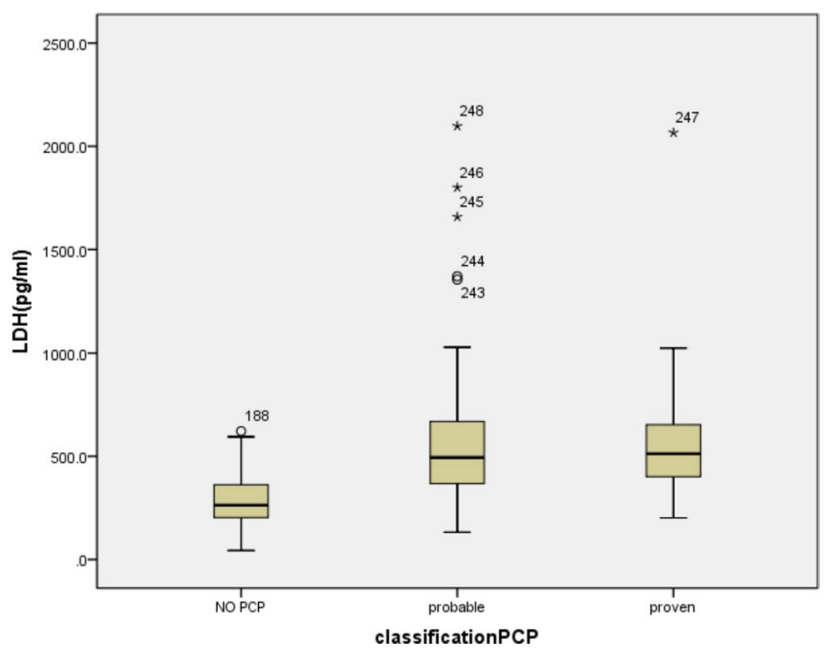

Figure 3. (Box-and-whisker plot): The mean levels of LDH in the no PCP, probable PCP and proven PCP groups were 297.62 U/L, 547.88 U/L, and 854.23 U/L. The medians of the 3 groups were $263.0(203.0,302.0) \mathrm{pg} /$ $\mathrm{mL}, 494.0(367.0,672.0) \mathrm{pg} / \mathrm{mL}, 512.0(401.0,653.0) \mathrm{pg} / \mathrm{mL}$ respectively. 


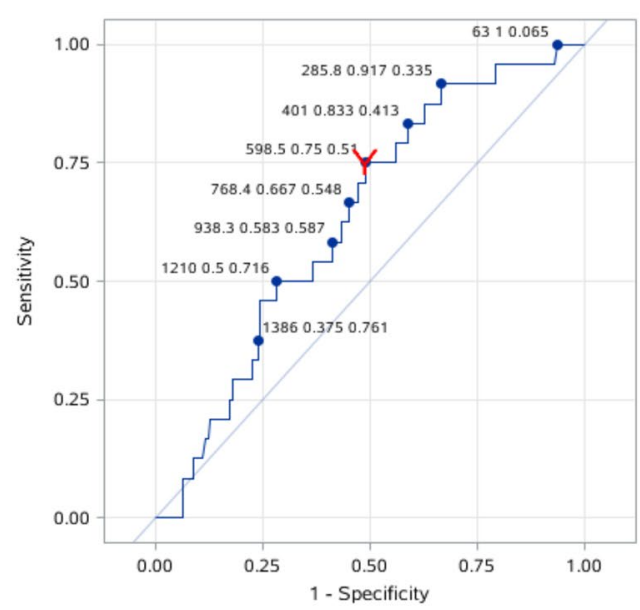

Points labeled by: BG_sens__spec_.

C

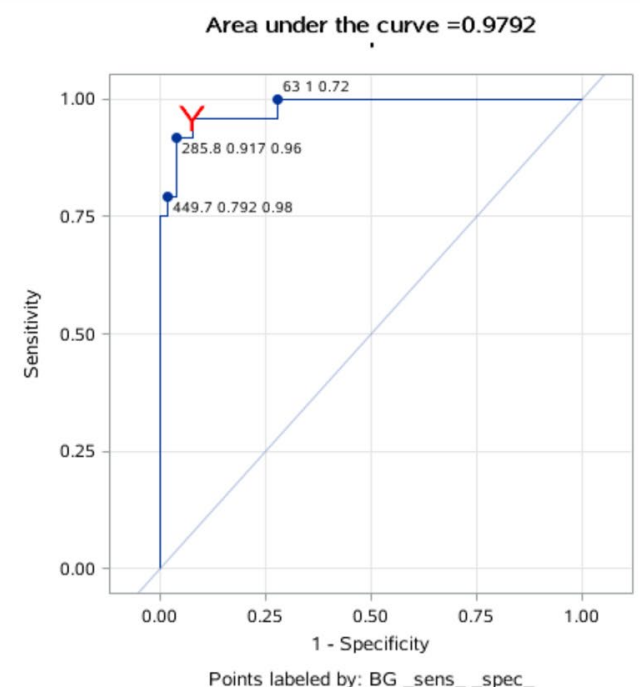

b

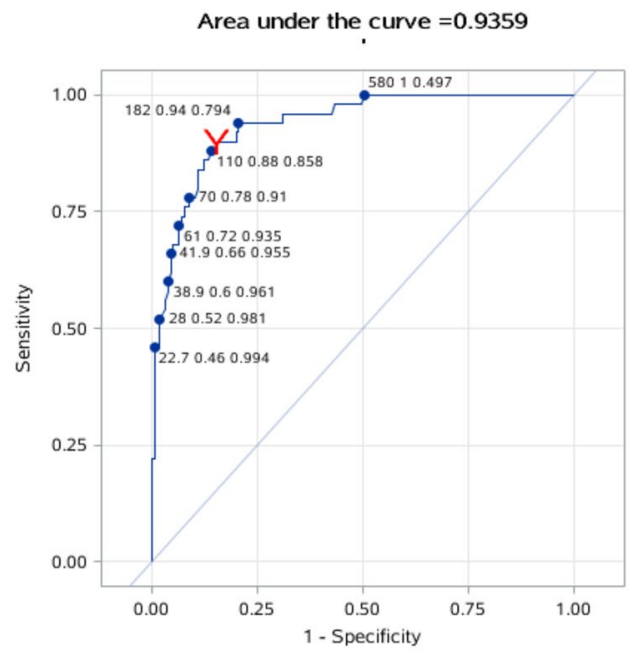

Points labeled by: BG_sens__spec_

d

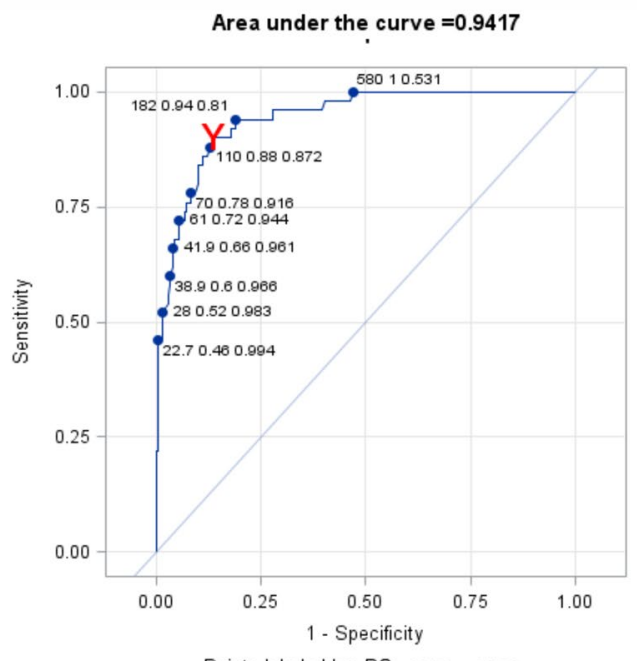

Points labeled by: BG_sens__spec_

Figure 4. (a) The ROC curves for BG of probable PCP and proven PCP groups; (b) The ROC curves for BG of probable PCP and NO PCP groups; (c) The ROC curves for BG of proven PCP and NO PCP groups; (d) The ROC curves for BG of proven/probable PCP and NO PCP groups.

\section{Discussion}

To our knowledge, the definitive diagnosis of PCP is still challenging. Most importantly, the organism cannot be cultured in clinical laboratories, so the diagnosis depends on examination of respiratory secretions usually obtained by invasive bronchoscopy, and visualization of the organism in respiratory secretions requires special staining techniques ${ }^{19}$. Beta-glucan and lactate dehydrogenase, as non-invasive markers, can provide useful, noninvasive adjunctive diagnostic tools. In this retrospective observational study, the usefulness of serum BG and $\mathrm{LDH}$ in the diagnosis of PCP in non-HIV patients was assessed. The results supported that these two biomarkers have excellent power for discriminating proven PCP or probable PCP from non-PCP patients. Serum BG and LDH tests may be incorporated in the diagnosis of PCP in non-HIV immunocompromised patients with fever, dyspnoea and infiltrates on chest CT scans.

Several previous publications have studied BG as a diagnostic tool for PCP in immunocompromised patients. We reviewed the recent literature on serum BG as a marker for PCP (Table 3) 3,5-7,9-11,13,20-29, and there have been few studies on non-HIV patients. Mark et al. summarized studies before 2009 and found that elevated serum BG was a reliable indicator for the diagnosis of PCP in solid organ transplantation and haematological malignancy patients ${ }^{8}$. In 2019, Sejal evaluated 53 cases of almost the same population and came to the same conclusion ${ }^{14}$. Some authors mentioned that the burden of $P$. jirovecii organisms in the lungs of HIV PCP patients is usually heavier than that in non-HIV PCP patients ${ }^{30}$; thus, we studied the reliability of the BG test for the diagnosis of $\mathrm{PCP}$ in non-HIV immunocompromised patients. In our study, patients' underlying conditions mainly included rheumatic immune diseases, interstitial lung diseases and glomerulonephritis without a definitive cause, and $\mathrm{BG}$ was also a reliable indicator for PCP. 
a

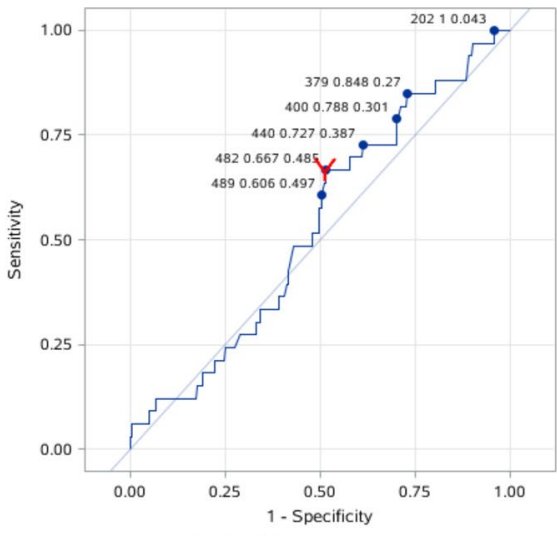

Points labeled by: LDH_sens__spec

C

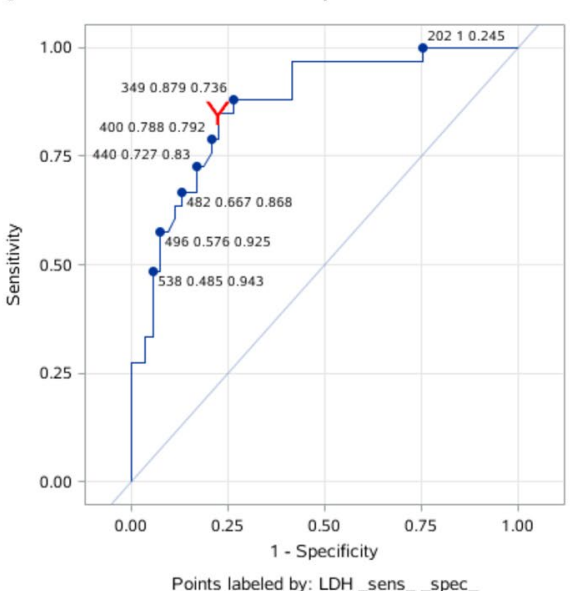

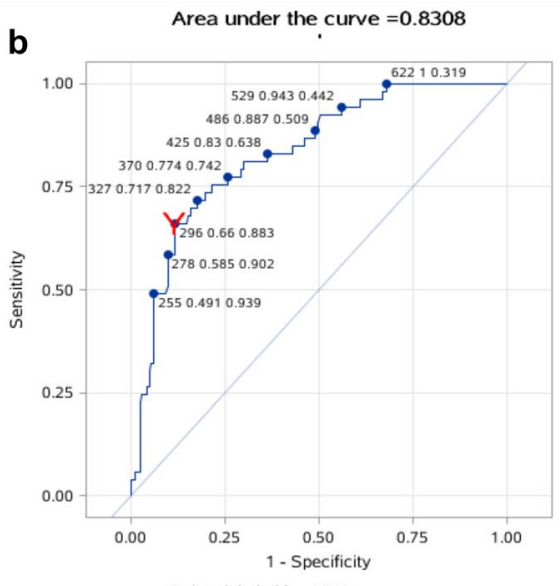

Points labeled by: LDH_sens__spec_

d

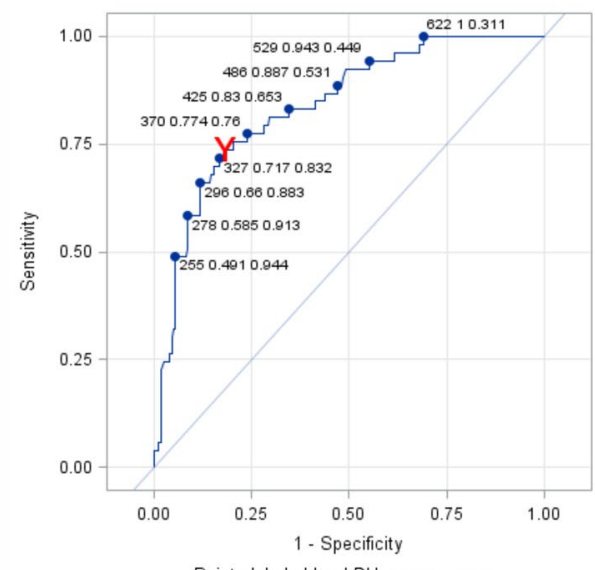

Points labeled by: LDH_sens__spec

Figure 5. (a) The ROC curves for LDH of probable PCP and proven PCP groups; (b) The ROC curves for LDH of probable PCP and NO PCP groups; (c) The ROC curves for LDH of proven PCP and NO PCP groups; (d) The ROC curves for LDH of proven/probable PCP and NO PCP groups.

In studies of HIV-positive patients, the cut-off levels of BG were $80 \mathrm{pg} / \mathrm{mL}, 200 \mathrm{pg} / \mathrm{mL}, 300 \mathrm{pg} / \mathrm{ml}$ and $400 \mathrm{pg} /$ $\mathrm{mL}^{6,7,9,21}$. The cut-off values in non-HIV patient studies were also volatile ${ }^{8,20,24}$; in this study, the cut-off value of BG was $110 \mathrm{pg} / \mathrm{mL}$. As mentioned before, we suspected that the difference was due to the different numbers of $P$. jirovecii organisms in different patients. However, J. Held proved that BG did not correlate with the $P$. jirovecii burden ${ }^{25}$. More data and research are required for a precise range of BG levels to diagnose PCP.

There were 23 false-positive cases in this study, and the reasons are chiefly as follows: the main reason is that beta-glucan is not a species-specific marker for P. jirovecii, so the BG test in the care of immunocompromised pneumonia is "pan-fungal" in nature. Some invasive fungal infections, including candidaemia, aspergillosis, fusariosis, trichosporiosis, and histoplasmosis, may trigger a high level of $\mathrm{BG}^{27}$. Although we excluded patients with certain non-PCP fungal infections, on review, 9 patients were treated with nystatin for oral candidiasis, and 4 patients received voriconazole as an empirical therapy without clear evidence of fungal infection. Immunocompromised patients are at high risk of fungal infection, so occult fungal infection may affect the results. Although several studies have shown that the serum BG levels of patients with invasive fungal infections are generally lower than those with $\mathrm{PCP}^{13,24}$, when these two types of opportunistic infections are mixed, they are difficult to identify. In addition, false-positive BG results can occur in patients on haemodialysis, with gram-negative bacteraemia, severe mucositis, or with the use of some antibiotics and intravenous immunoglobulins ${ }^{3,31}$. In our cases, the only factor was the use of antimicrobials, which mainly included third-generation cephalosporins, carbapenems and cefoperazone sulbactam; however, the effects of these antibiotics on serum BG are still unclear. On the other hand, 31 cases were false negatives. The BG test was performed in the early stage of PCP in which few P. jirovecii cysts were destroyed, and thus, the BG level did not increase. One-third of these 31 patients were taking a preventive dose of sulfanilamide for PCP; sulfanilamide prevention may also reduce the level of the BG test.

Studies on the accuracy of LDH in the diagnosis of PCP are rare ${ }^{6,13,17,20}$. Deng C performed a systematic review and concluded that the sensitivity of $\mathrm{LDH}$ in the diagnosis of $\mathrm{PCP}$ is above $80 \%$, and the value is especially high in the AIDS group, but the specificities varied greatly, from 6 to $85 \%{ }^{17}$. Our study was the first aimed at non-HIV immunocompromised patients, and the sensitivity and specificity were meaningful. There were still 


\begin{tabular}{|c|c|c|c|c|}
\hline 1st Author & Year & Journal & N (PCP+) HIV+/HIV- & Main result \\
\hline $\operatorname{Maria}^{5}$ & 2009 & Clin Vaccine Immunol & $23 / 8$ & $\begin{array}{l}\text { The medium BG value of PCP and NPCP group were } 423 \mathrm{pg} / \mathrm{mL} \text { and } 60 \mathrm{pg} / \mathrm{mL} \\
\text { respectively }\end{array}$ \\
\hline Esteves ${ }^{6}$ & 2014 & Eur J Clin Microbiol Infect Dis & $100 / 0$ & $\begin{array}{l}\text { The most promising cut-off levels for diagnosis of PCP were determined to be } 400 \mathrm{pg} \text { / } \\
\text { mL of BG and } 350 \mathrm{U} / \mathrm{L} \text { of } \mathrm{LDH} \text {, which combined with clinical data presented } 92.8 \% \\
\text { sensitivity, } 83.9 \% \text { specificity }\end{array}$ \\
\hline Helena $^{7}$ & 2019 & BMC Infect Dis & $21 / 0$ & $\begin{array}{l}\text { Beta glucan with cut-off level } 200 \mathrm{pg} / \mathrm{mL} \text { combined with a positive Pneumocystis } \\
\text { jirovecii PCR result had sensitivity and specificity of } 92 \text { and } 90 \% \text {, respectively in PCP } \\
\text { diagnosis }\end{array}$ \\
\hline $\operatorname{Mark}^{8}$ & 2011 & J Infection & $0 / 21$ & $\begin{array}{l}\text { Elevated serum b-D-glucan was a reliable indicator for PCP with a sensitivity of } 0.90 \\
\text { and specificity of } 0.89 \text { at the } 60 \mathrm{pg} / \mathrm{mL} \text { cut-off }\end{array}$ \\
\hline Salerno $^{9}$ & 2014 & Respir Med & $119 / 0$ & $\begin{array}{l}\text { BG yielded a sensitivity of } 91 \% \text { and specificity of } 92 \% \text { for PCP diagnosis at a } 300 \mathrm{pg} / \mathrm{mL} \\
\text { cut-off level }\end{array}$ \\
\hline Engsbro $^{12}$ & 2019 & Med Mycol & NA & The Sensitivity and specificity were $87 \%$ and $70 \%$ when the level of BG was $95 \mathrm{pg} / \mathrm{mL}$ \\
\hline Esteves $^{13}$ & 2015 & Clin Microbiol Infect & $89 / 34$ & $\begin{array}{l}\text { BG was found to be the most reliable serologic biomarker for PCP diagnosis with } \\
\text { sensitivity } 91.1 \% \text {, Specificity } 71.6 \% \text {, followed by KL-6, LDH and SAM. The BG/KL-6 } \\
\text { combination test was the most accurate serologic approach for PCP diagnosis, with } \\
94.3 \% \text { sensitivity and } 89.6 \% \text { specificity }\end{array}$ \\
\hline Sejal $^{14}$ & 2019 & Clin infect dis & $0 / 53$ & $\begin{array}{l}\text { With PCP PCR alone as the reference method, BG }(\geq 80 \mathrm{pg} / \mathrm{mL}) \text { had a sensitivity of } \\
69.8 \% \text { and a specificity of } 81.2 \% \text { for PCP. At } \geq 200 \mathrm{pg} / \mathrm{mL} \text {, in patients with a positive } \\
\text { PCR and a compatible PCP clinical syndrome, BDG had a sensitivity and a specificity } \\
\text { of } 70 \% \text { and } 100 \%\end{array}$ \\
\hline Akira $^{21}$ & 2011 & J Clin Microbiol & - & $\begin{array}{l}\text { This meta-analysis of twelve studies for PCP from January } 1966 \text { to November } 2010 \\
\text { showed BG had had a sensitivity of } 96 \% \text { and a specificity of } 84 \% \text { for PCP }\end{array}$ \\
\hline Brian $\mathrm{R}^{22}$ & 2013 & AIDS & $129 / 0$ & $\begin{array}{l}\text { The sensitivity of BG for PCP in HIV-participants with respiratory symptoms was } \\
92.8 \%(95 \% \text { CI } 87.2-96.5 \%) \text {, and specificity } 75.0 \%(50.9-91.3 \%) \text { at the cutoff of } 80 \mathrm{pg} / \\
\mathrm{mL}\end{array}$ \\
\hline Karageorgopoulos ${ }^{23}$ & 2013 & Clin Microbiol Infect & - & $\begin{array}{l}\text { Fourteen studies were included in the meta-analysis. BG data were analysed for } \\
357 \text { PCP cases and } 1723 \text { controls. The average sensitivity and specificity of BG were } \\
94.8 \% \text { and } 86.3 \% \text {, respectively }\end{array}$ \\
\hline Hyo-Ju ${ }^{24}$ & 2017 & Plos ONE & $0 / 50$ & $\begin{array}{l}\text { The mean } \pm \text { SD of the concentration of BG in the patients with PCP were similar to } \\
\text { those of patients with candidemia and chronic disseminated candidiasis, but higher } \\
\text { than those of patients with invasive aspergillosis, mucormycosis and tuberculosis as well } \\
\text { as healthy volunteers }\end{array}$ \\
\hline J. Held ${ }^{25}$ & 2011 & Clin Microbiol Infect & $17 / 33$ & $\begin{array}{l}\text { Serum BG is an excellent diagnostic performance with a sensitivity of } 98.0 \% \text { and a } \\
\text { specificity of } 94 \% \text {. BG did not correlate with the outcome of patients or with the } P \text {. } \\
\text { jirovecii burden }\end{array}$ \\
\hline $\operatorname{Karl}^{26}$ & 2018 & J Clin Microbiol & $36 / 74$ & $\begin{array}{l}\text { BG identified } 66 / 73 \text { patients with positive qPCR tests for an overall sensitivity of } 91 \% \text {, } \\
\text { and it was negative in } 25 / 25 \text { controls with negative qPCR for a specificity of } 100 \% \text { using } \\
\text { the predefined cut-off } 11-\mathrm{pg} / \mathrm{mL}\end{array}$ \\
\hline $\mathrm{Paul}^{27}$ & 2011 & Clin Infect Dis & $173 / 0$ & $\begin{array}{l}\text { The sensitivity of b-glucan dichotomized at } 80 \mathrm{pg} / \mathrm{mL} \text { for the diagnosis of PCP was } 92 \% \\
\text { (95\% confidence interval [CI] 87-96\%), and the specificity was } 65 \%\end{array}$ \\
\hline Tamayo $^{28}$ & 2009 & Clin Infect Dis & $111 / 0$ & $\begin{array}{l}\text { A b-d-glucan cut-off value of } 23.2 \mathrm{pg} / \mathrm{mL} \text { had a sensitivity of } 96.4 \% \text { and a specificity of } \\
87.8 \% \text { in PCP diagnosis }\end{array}$ \\
\hline Wei-Jie ${ }^{29}$ & 2015 & J Thorac Dis & - & $\begin{array}{l}\text { The meta-analysis of } 13 \text { studies estimates for serum-BG assay for definite PCP were as } \\
\text { follows: Se } 0.91 \text { [ } 95 \% \text { confidence interval (CI) } 0.88-0.93 \text { ]; Sp } 0.75 \text { ( } 95 \% \text { CI } 0.68-0.81 \text { ) }\end{array}$ \\
\hline
\end{tabular}

Table 3. An overview of recent literatures on serum BG as a marker for PCP.

31 false-negative cases and 23 false-positive cases. We reviewed the false-negative cases and found that LDH was tested in the early stage of the disease when patients had a low $P$. jirovecii burden. The majority of the LDH values were close to the cut-off value $(20 / 31$ of the $\mathrm{LDH}$ levels were over $200 \mathrm{U} / \mathrm{L}$, which is close to the cut-off value of 296 U/L). In cases of false positives, although we excluded cases with abnormal liver function and haematological malignancies, LDH is widely expressed in human tissues and is released when the cytoplasmic cell membrane is damaged. The LDH result could be influenced by other lung infections and various extrapulmonary disorders.

To our knowledge, this was the largest study of a novel diagnostic test for PCP among non-HIV-infected individuals. Since popularizing BG and LDH testing, we have found that the yield of PCP diagnoses has increased, while empirically treated cases and the need for bronchoscopy have been reduced. We hope that our study results can help BG and LDH become helpful adjunctive tests for PCP diagnosis in non-HIV immunocompromised patients.

At the same time, the study has some limitations. First, the sensitivity and specificity of BG and LDH to distinguish proven PCP from probable PCP are poor. Second, we did not analyse the relationship between the serum marker level and the $P$. jirovecii burden, so the response to therapy based on BG or LDH should be evaluated. Third, cases were excluded based on the criteria given above, but this method may overemphasize the specificity of the tests; unfortunately, the sample sizes of patients who both matched the exclusion criteria and took the BG or LDH test were small, and we did not analyse the influence of these factors. Finally, because of the different inclusion and exclusion criteria of LDH and BG group, the target populations are also different. The sample size of population meeting the inclusion and exclusion criteria of these two groups was too small to analyse, there is no combined analysis for both BG and LDH in this study. Furtherly we could collect enough cases to analyse 
the BG level, LDH level and even more related diagnostic tests comprehensively, in this way, the PCP might be recognized more specifically and sensitively in the immunocompromised patients.

In summary, we found that in HIV-uninfected immunocompromised patients with pneumonia symptoms, positive BG and $\mathrm{LDH}$ tests had a high predictive value for the diagnosis of PCP. We suggest that serum BG and LDH levels can provide helpful diagnostic support for PCP in this population, especially in settings where invasive testing for PCP is either unavailable or expensive.

\section{Data availability}

The datasets used and analysed during the current study are available from the corresponding author on reasonable request.

\section{Code availability}

11578.

Received: 28 September 2020; Accepted: 16 April 2021

Published online: 29 April 2021

\section{References}

1. Thomas, C. F. Jr. \& Limper, A. H. Pneumocystis pneumonia. N. Engl. J. Med. 350(24), 2487-2498 (2004).

2. Limper, A. H., Adenis, A., Le, T. \& Harrison, T. S. Fungal infections in HIV/AIDS. Lancet. Infect. Dis. 17(11), e334-e343 (2017).

3. Oladele, R. O., Otu, A. A., Richardson, M. D. \& Denning, D. W. Diagnosis and management of pneumocystis pneumonia in resource-poor settings. J. Health Care Poor Underserved 29(1), 107-158 (2018).

4. Song, Y., Ren, Y., Wang, X. \& Li, R. Recent advances in the diagnosis of pneumocystis pneumonia. Med. Mycol. J. 57(4), E111-e116 (2016).

5. Cuétara, M. S. et al. Detection of (1->3)-beta-D-glucan as an adjunct to diagnosis in a mixed population with uncommon proven invasive fungal diseases or with an unusual clinical presentation. Clin. Vaccine Immunol. CVI 16(3), 423-426 (2009).

6. Esteves, F. et al. (1-3)-beta-D-glucan in association with lactate dehydrogenase as biomarkers of Pneumocystis pneumonia (PcP) in HIV-infected patients. Eur. J. Clin. Microbiol. Infect. Dis. 33(7), 1173-1180 (2014).

7. Hammarström, H. et al. Serum-based diagnosis of Pneumocystis pneumonia by detection of Pneumocystis jirovecii DNA and 1,3- $\beta$-D-glucan in HIV-infected patients: A retrospective case control study. BMC Infect. Dis. 19(1), 658 (2019).

8. de Boer, M. G. et al. $\beta$-D-glucan and S-adenosylmethionine serum levels for the diagnosis of Pneumocystis pneumonia in HIVnegative patients: A prospective study. J. Infect. 62(1), 93-100 (2011).

9. Salerno, D. et al. Serum and bal beta-D-glucan for the diagnosis of Pneumocystis pneumonia in HIV positive patients. Respir. Med. 108(11), 1688-1695 (2014).

10. Matsumura, Y. et al. Pneumocystis polymerase chain reaction and blood $(1 \rightarrow 3)$ - $\beta$-D-glucan assays to predict survival with suspected Pneumocystis jirovecii pneumonia. J. Infect. Chemother. 20(2), 109-114 (2014).

11. White, P. L. et al. An evaluation of the performance of the Dynamiker Fungus (1-3)- $\beta$-D-Glucan Assay to assist in the diagnosis of Pneumocystis pneumonia. Med. Mycol. 56(6), 778-781 (2018).

12. Engsbro, A. L., Najat, S., Jørgensen, K. M., Kurtzhals, J. A. L. \& Arendrup, M. C. Diagnostic accuracy of the 1,3- $\beta$-D-glucan test for pneumocystis pneumonia in a tertiary university hospital in Denmark: A retrospective study. Med. Mycol. 57(6), 710-717 (2019).

13. Esteves, F. et al. Diagnosis of Pneumocystis pneumonia: Evaluation of four serologic biomarkers. Clin. Microbiol. Infect. 21(4), 379.e371-310 (2015).

14. Morjaria, S. et al. Clinical performance of $(1,3)$ Beta-D Glucan for the diagnosis of pneumocystis pneumonia (PCP) in cancer patients tested with PCP polymerase chain reaction. Clin. Infect. Dis. 69(8), 1303-1309 (2019).

15. Matsumura, Y. et al. Clinical characteristics of Pneumocystis pneumonia in non-HIV patients and prognostic factors including microbiological genotypes. BMC Infect. Dis. 11, 76 (2011).

16. Fujii, T., Nakamura, T. \& Iwamoto, A. Pneumocystis pneumonia in patients with HIV infection. Clinical manifestations, laboratory findings, and radiological features. J. Infect. Chemother. 13(1), 1-7 (2007).

17. Deng, C., Li, Y. \& Li, Y. Systemic review: The accuracy of lactic dehydrogenase in the diagnosis of pneumocystis pneumonia. Zhonghua wei zhong bing ji jiu yi xue 30(4), 322-326 (2018).

18. Wenjing, L. et al. Evaluation of real-time PCR and Grocott-Methenamine silver stain for diagnosing of Pneumocystis pneumonia. J. Mod. Lab. 32(5), 28-31 (2017).

19. Kovacs, J. A., Gill, V. J., Meshnick, S. \& Masur, H. New insights into transmission, diagnosis, and drug treatment of Pneumocystis carinii pneumonia. JAMA 286(19), 2450-2460 (2001).

20. Morjaria, S. et al. Clinical. Performance of $(1,3)$ Beta-D-Glucan for the diagnosis of Pneumocystis pneumonia in cancer patients tested with PCP PCR. Clin. Infect. Dis. 69(8), 1303-1309 (2019).

21. Onishi, A. et al. Diagnostic accuracy of serum 1,3- $\beta$-D-glucan for pneumocystis jiroveci pneumonia, invasive candidiasis, and invasive aspergillosis: Systematic review and meta-analysis. J. Clin. Microbiol. 50(1), 7-15 (2012).

22. Wood, B. R. et al. Test performance of blood beta-glucan for Pneumocystis jirovecii pneumonia in patients with AIDS and respiratory symptoms. AIDS (London, England) 27(6), 967-972 (2013).

23. Karageorgopoulos, D. E. et al. Accuracy of $\beta$-D-glucan for the diagnosis of Pneumocystis jirovecii pneumonia: A meta-analysis. Clin. Microbiol. Infect. 19(1), 39-49 (2013).

24. Son, H. J. et al. Diagnostic performance of the (1-3)- $\beta$-D-glucan assay in patients with Pneumocystis jirovecii compared with those with candidiasis, aspergillosis, mucormycosis, and tuberculosis, and healthy volunteers. PLoS ONE 12(11), e0188860 (2017).

25. Held, J., Koch, M. S., Reischl, U., Danner, T. \& Serr, A. Serum $(1 \rightarrow 3)$ - $\beta$-D-glucan measurement as an early indicator of Pneumocystis jirovecii pneumonia and evaluation of its prognostic value. Clin. Microbiol. Infect. 17(4), 595-602 (2011).

26. Dichtl, K., Seybold, U. \& Wagener, J. Evaluation of a turbidimetric $\beta$-d-Glucan test for detection of Pneumocystis jirovecii pneumonia. J. Clin. Microbiol. 56(7), e00286 (2018).

27. Sax, P. E. et al. Blood (1->3)-beta-D-glucan as a diagnostic test for HIV-related Pneumocystis jirovecii pneumonia. Clin. Infect. Dis. 53(2), 197-202 (2011).

28. Watanabe, T. et al. Serum (1->3) beta-D-glucan as a noninvasive adjunct marker for the diagnosis of Pneumocystis pneumonia in patients with AIDS. Clin. Infect. Dis. 49(7), 1128-1131 (2009).

29. Li, W. J., Guo, Y. L., Liu, T. J., Wang, K. \& Kong, J. L. Diagnosis of pneumocystis pneumonia using serum (1-3)- $\beta$-D-Glucan: A bivariate meta-analysis and systematic review. J. Thorac. Dis. 7(12), 2214-2225 (2015).

30. Desmet, S. et al. Serum (1-3)-beta-D-glucan as a tool for diagnosis of Pneumocystis jirovecii pneumonia in patients with human immunodeficiency virus infection or hematological malignancy. J. Clin. Microbiol. 47(12), 3871-3874 (2009). 
31. White, P. L., Backx, M. \& Barnes, R. A. Diagnosis and management of Pneumocystis jirovecii infection. Expert Rev. Anti Infect. Ther. 15(5), 435-447 (2017).

\section{Author contributions}

All authors contributed to the study conception and design. Date collection and description were performed by R.X.S. and D.L. The first draft of the manuscript was written by R.X.S. and J.Y., all authors commented on previous versions of the manuscript. All authors read and approved the final manuscript.

\section{Competing interests}

The authors declare no competing interests.

\section{Additional information}

Supplementary Information The online version contains supplementary material available at https:/doi.org/ 10.1038/s41598-021-88729-z.

Correspondence and requests for materials should be addressed to J.Y.

Reprints and permissions information is available at www.nature.com/reprints.

Publisher's note Springer Nature remains neutral with regard to jurisdictional claims in published maps and institutional affiliations.

(c) (i) Open Access This article is licensed under a Creative Commons Attribution 4.0 International

License, which permits use, sharing, adaptation, distribution and reproduction in any medium or format, as long as you give appropriate credit to the original author(s) and the source, provide a link to the Creative Commons licence, and indicate if changes were made. The images or other third party material in this article are included in the article's Creative Commons licence, unless indicated otherwise in a credit line to the material. If material is not included in the article's Creative Commons licence and your intended use is not permitted by statutory regulation or exceeds the permitted use, you will need to obtain permission directly from the copyright holder. To view a copy of this licence, visit http://creativecommons.org/licenses/by/4.0/.

(C) The Author(s) 2021 